\title{
Clinical presentation and management of lacrimal gland tumours
}

\author{
JOHN E. WRIGHT, WILLIAM B. STEWART, ${ }^{1}$ AND GREGORY B. KROHEL ${ }^{2}$ \\ From the Orbital Clinic, Moorfields Eye Hospital, City Road, London
}

SUMMARY This paper presents the clinical and pathological findings in 40 consecutive patients seen with primary tumours arising from the lacrimal gland. Twenty patients had a benign mixedcell tumour. They presented in a recognisable clinical manner with a painless mass in the region of the lacrimal gland which slowly enlarged over a period of at least 1 year before consultation. Twenty patients had a carcinoma of the lacrimal gland. They had a short history and experienced pain. On clinical grounds they could not be distinguished from inflammatory lesions in the region of the lacrimal gland. The method of treating these 2 groups of patients is described and methods of dealing logically with their problems are suggested.

This communication presents an analysis of the mode of clinical presentation of a large series of lacrimal gland tumours. The correct management of lacrimal fossa lesions depends on recognising the characteristic clinical presentation of benign mixed cell tumours so that a biopsy is avoided and the capsule of the tumour remains intact. Excision of the whole of the lacrimal gland together with the tumour avoids any risk of seeding tumour cells into the adjacent tissues with the possibility of a recurrence with malignant potential (Rubin, 1947; Zimmerman et al., 1962; Waller et al., 1973).

All other lesions in this region, apart from acute dacryoadenitis and dermoid cysts, require incisional biopsy through an anterior approach so that a definitive histological diagnosis can be made. After such a biopsy histological as well as clinical criteria can be used to decide which type of treatment is most appropriate.

\section{Material and methods}

Clinical records and histopathological findings of 108 patients with lesions of the fossa of the lacrimal gland seen in the Orbital Clinic at Moorfields Eye Hospital, London, from 1968 to 1979 were reviewed

\footnotetext{
${ }^{1}$ Department of Ophthalmology, Pacific Medical Center, San Francisco, California, USA.

${ }^{2}$ Neuro-Ophthalmology Service, Department of Ophthalmology, Albany Medical College, New York, USA.
}

Correspondence to John E. Wright, FRCS, Moorfields Eye Hospital, City Road, London ECIV 2PD. in an attempt to identify clinical characteristics that would allow recognition of benign mixed lacrimal gland tumours from other lesions of the superotemporal orbit. There were 40 cases of benign or malignant epithelial lacrimal gland tumours. All had plain radiographs and a histological diagnosis. The other cases were nonepithelial lesions such as acute and chronic nongranulomatous inflammation, granulomatous inflammation, ideopathic inflammatory pseudotumour, lymphoma, and dermoid cysts. Orbital involvement in some of these cases was not confined to the lacrimal fossa.

Evaluation of patients presenting with lacrimal gland fossa symptoms and signs included a general medical examination, blood studies, and plain and tomographic radiographs of the orbits. Orbital ultrasonography and computerised tomography were performed in selected cases.

\section{Results}

Twenty patients in the series had benign mixed lacrimal gland tumours. They comprised 11 men and 9 women, with an age range of 21-68 years (Table 1). The duration of symptoms was greater than 1 year in 19 patients, only 1 patient with a benign mixed tumour had pain. Radiological abnormalities were seen in 18 patients; all had enlargement of the lacrimal gland fossa due to pressure erosion of the overlying bone. Occasionally this erosion was sufficient to breach the orbital wall, with exposure of the dura of the anterior cranial fossa. In addition sclerosis of bone was 
Table 1 Clinical details of 40 patients with lacrimal gland tumours

\begin{tabular}{|c|c|c|c|c|}
\hline \multirow[b]{2}{*}{$\begin{array}{l}\text { Age } \\
\text { (years) }\end{array}$} & \multirow{2}{*}{$\begin{array}{l}\text { Benign } \\
\text { mixed cell } \\
\text { lacrimal } \\
\text { gland } \\
\text { tumours } \\
(20)\end{array}$} & \multicolumn{3}{|c|}{ Malignant epithelial tumours (20) } \\
\hline & & $\begin{array}{l}\text { Malignant } \\
\text { mixed cell } \\
\text { tumours } \\
(2)\end{array}$ & $\begin{array}{l}\text { Adenocystic } \\
\text { carcinomas } \\
(9)\end{array}$ & $\begin{array}{l}\text { Other } \\
\text { carcinomas } \\
(9)\end{array}$ \\
\hline $0-$ & - & 1 & 2 & 1 \\
\hline $20-$ & 3 & 1 & 1 & 1 \\
\hline $30-$ & 5 & - & 2 & - \\
\hline $40-$ & 6 & - & 1 & 1 \\
\hline $50-$ & 5 & - & 2 & 2 \\
\hline $60-$ & 1 & - & 1 & 4 \\
\hline $70 \div$ & - & - & - & - \\
\hline \multicolumn{5}{|l|}{ Sex } \\
\hline $\mathbf{M}$ & 11 & - & 4 & 5 \\
\hline $\mathrm{F}$ & 9 & 2 & 5 & 4 \\
\hline \multicolumn{5}{|l|}{$\begin{array}{l}\text { Duration of } \\
\text { symptoms } \\
\text { (months) }\end{array}$} \\
\hline $0-$ & - & - & - & - \\
\hline $3-$ & - & 1 & 3 & 3 \\
\hline $6-$ & - & - & 1 & 2 \\
\hline 9- & 1 & - & 2 & 3 \\
\hline $12-$ & 3 & - & 2 & 1 \\
\hline $18-$ & 16 & 1 & 1 & - \\
\hline \multicolumn{5}{|l|}{ Pain } \\
\hline Present & 1 & 1 & 8 & 6 \\
\hline Not present & 19 & 1 & 1 & 3 \\
\hline \multicolumn{5}{|c|}{$\begin{array}{l}\text { X-ray findings } \\
\text { (plain and } \\
\text { tomographic) }\end{array}$} \\
\hline Normal & 2 & - & 2 & 1 \\
\hline Pressure & 18 & 2 & 6 & 5 \\
\hline Destruction & - & - & 1 & 3 \\
\hline
\end{tabular}

present in 1 patient in association with pressure changes.

Malignant neoplasms accounted for 20 of the 40 patients with primary epithelial tumours originating in the lacrimal gland. There were 9 adenoid cystic carcinomas, 2 malignant mixed cell tumours, and 9 carcinomas of other types. There was a wide age distribution. It was noteworthy that 7 patients were under 30 years of age, and 4 of the 7 had been treated elsewhere for suspected orbital cellulitis. Fifteen patients had had symptoms for less than 1 year. Only 1 of the patients with a history of longer than 12 months had an operable tumour. This was a case of carcinomatous change in a pre-existing benign mixed tumour. Fifteen patients had noticed pain, and 3 of these patients had associated loss of sensation in the distribution of the frontal nerve. Radiological changes were encountered in 17 patients. Thirteen showed a pressure erosion of overlying bone; 4 patients had demonstrable destructive changes in adjacent bone on plain or tomographic views. Of the 13 patients with pressure changes, 3 had calcification within the lacrimal gland, and 2 had sclerotic changes in the bone adjacent to the expanding lacrimal fossa.

Nine of the malignant neoplasms were considered inoperable at the time of presentation at Moorfields Eye Hospital, either because of extensive involvement of bone or evidence of spread beyond the limits of even the most extensive surgical approach. All the cases in which tumour had spread beyond potential surgical margins had an interval from their initial presentation to an outside ophthalmic service until referral and/or definitive tissue diagnosis of greater than 3 months. The average interval was 4 months. For those patients who had resectable tumours the interval was only 2 months.

The nonepithelial lesions in the review included acute dacryoadenitis, idiopathic inflammatory pseudotumours, and lymphomatous lesions. These lesions represent a broad and varied spectrum of clinical presentation. Ages ranged from the first to the eighth decades. The duration of symptoms was generally less than 6 months. Inflammatory signs and discomfort were relatively common. $X$-ray abnormalities were most unusual.

\section{Discussion}

It is essential when dealing with lesions of the lacrimal gland that a neoplasm arising from the epithelium of the gland is recognised as soon as possible. The survival of the patient depends on the correct choice of treatment for the lesion. Analysis of cases presented in this communication has shown that, while carcinomas of the lacrimal gland cannot be distinguished from other rapidly expanding lesions in this region other than by histological examination, benign mixed-cell tumours can be readily recognised on clinical grounds.

The pattern of presentation of benign mixed lacrimal gland tumours is characteristic. They present in patients in their late 20 s to early 60 s with a slowly progressive painless swelling in the upper lid without inflammatory symptoms or signs. A palpable mass is often in the upper temporal quadrant of the orbit (Fig. 1). A careful detailed history will reveal that the duration of symptoms is more than 12 months. Radiographs usually show enlargement of the lacrimal fossa without invasion of overlying bone (Fig. 2) (Jones and Pfeiffer, 1954; Newton, 1962; Lloyd, 1975). Careful tomographic study may disclose this characteristic change when 
plain $x$-rays are negative (this occurred in 1 patient in our series). Coronal computerised tomography (CT) scans will confirm the presence and extent of the tumour (Fig. 3). Although the clinical presentation of cases of benign mixed lacrimal gland tumours has been described in a number of excellent reviews (Forrest, 1954; Davies, 1954; Reese, 1956; Zimmerman et al., 1962; Henderson, 1973), the characteristic clinical presentation of these tumours has not been fully recognised.

Occasionally a benign mixed lacrimal gland tumour may present in a different way. The patient gives a short history, there is a readily palpable mass, and radiographs are normal. This tumour arises from the palpebral lobe of the lacrimal gland and produces a slowly enlarging mass in the outer third of the upper eyelid. In this situation the mass

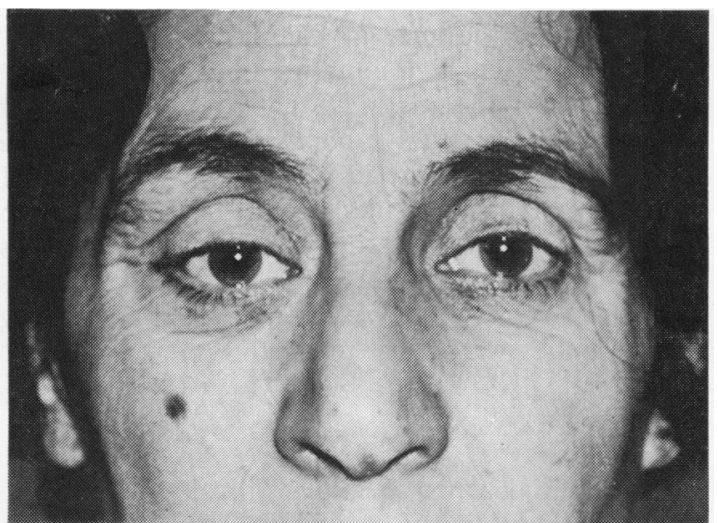

Fig. 1 Patient with benign mixed cell tumour in right orbit. Fullness of the right upper eyelid and proptosis noticed for 18 months

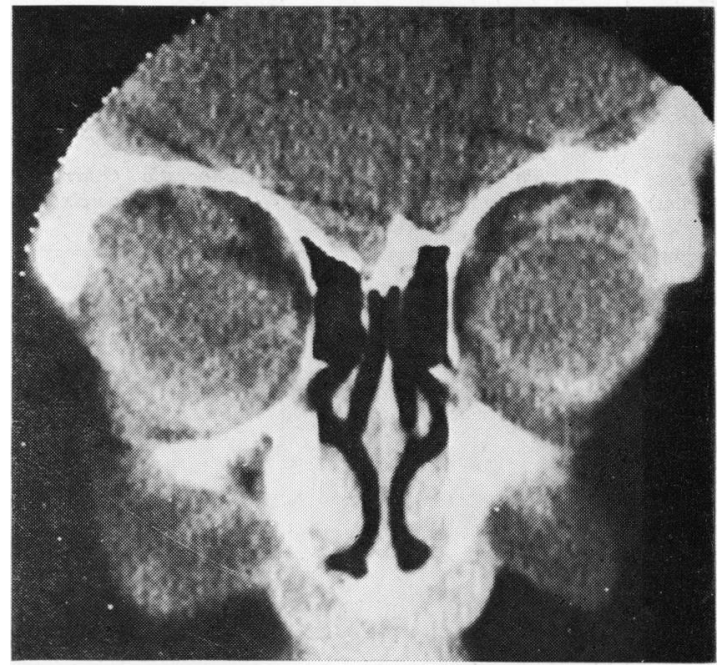

Fig. 3 Coronal CT scan demonstrating benign mixed cell tumour of the right lacrimal gland causing downward displacement of the globe

is noticed at an early stage, and because it lies anterior to the lacrimal fossa there is no bone erosion. Inevitably the mass is either biopsied or removed and the true nature of the lesion discovered by frozen section or routine histology. One such case is included in this communication.

Malignant tumours arising from the lacrimal gland in our series had 2 characteristic features-a short history with a rapidly worsening course and pain (Fig. 4). Both these features have been noted before. Two-thirds of the patients in Zimmerman's series with carcinoma unrelated to mixed tumours had symptoms of less than 9 months when they first

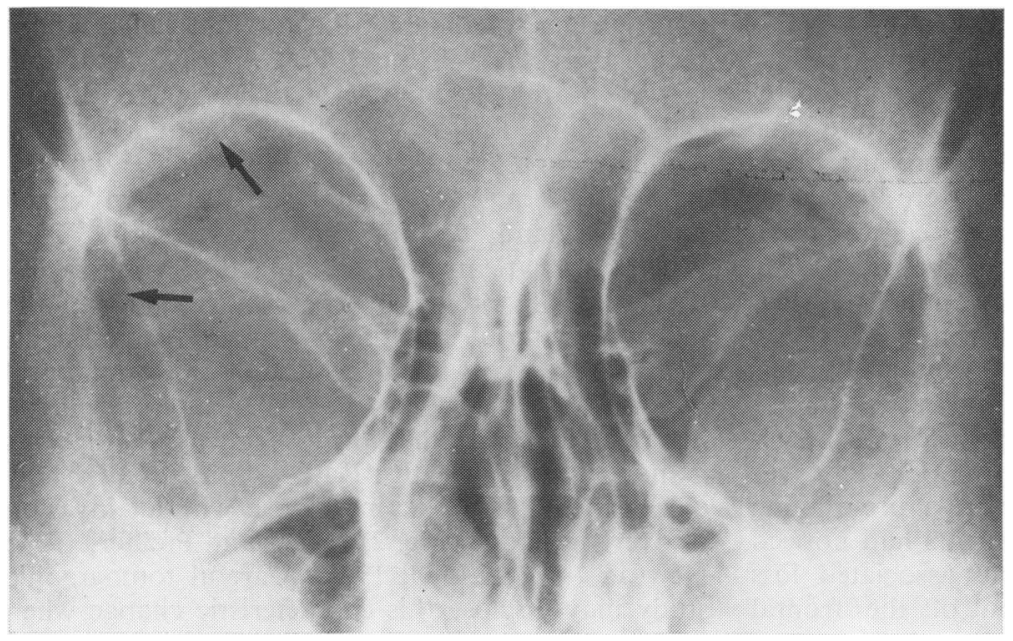

Fig. 2 Plain radiograph of orbits showing expansion of right lacrimal fossa (arrowed) produced by the slow growth of the benign mixed tumour 


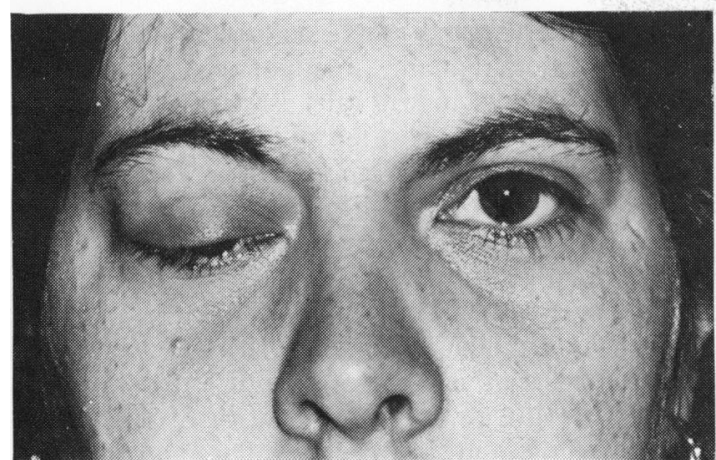

Fig. 4 Patient aged 31 with an adenoid cystic carcinoma. Painful mass for 6 months

presented with orbital complaints. The tendency for patients with malignant tumours to experience pain in a high percentage of patients was also noted by Zimmerman and Forrest. Radiographic findings may also help in distinguishing between the malignant and benign lacrimal gland tumour. Carcinomatous lesions often enlarge so rapidly that radiographs in the early stages are normal. Later, enlargement of the lacrimal fossa, with or without demonstrable invasion of bone, may be seen. In 3 patients calcification within the malignant tumour was demonstrated (Fig. 5). In all such patients it is important that both axial and coronal tomograms are obtained so that the structure of the bones in relation to the tumour can be examined in some detail. This type of assessment has now been supplemented by coronal and axial CT scans.

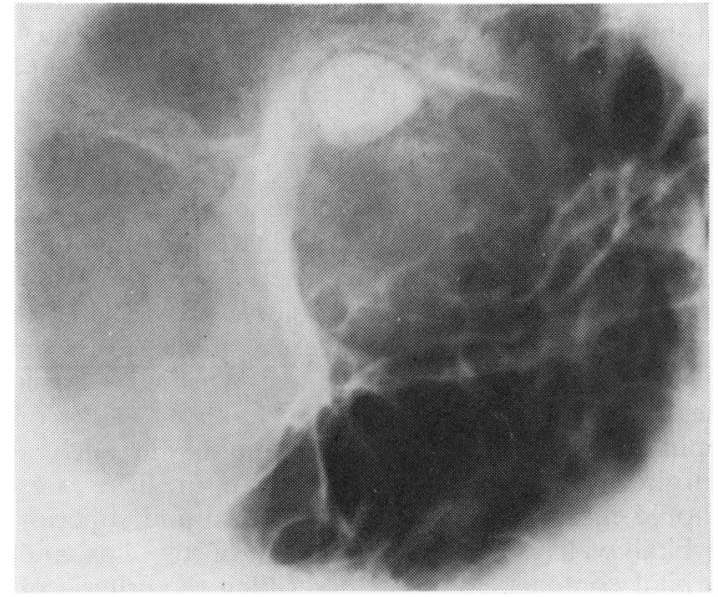

Fig. 5 Oblique radiograph of right orbit showing expansion of the lacrimal fossa by a partially calcified adenoid cystic carcinoma
Thus an overall picture of the extent of the lesion can be made.

Other lesions affecting the lacrimal fossa must be considered in the differential diagnosis. Acute dacryoadenitis, although described by Duke-Elder and MacFaul (1974) as a rare entity, occurs fairly frequently. Patients present with a short history measured in days rather than months. The upper lid is red, swollen, and extremely tender, and there is usually some conjunctival chemosis with an associated purulent discharge. The preauricular and cervical nodes are often enlarged and the patient pyrexial. Radiographs are invariably normal, and blood studies frequently reveal a raised white cell count. This type of dacryoadenitis should settle rapidly with appropriate systemic antibiotic therapy. Secondary dacryoadenitis related to mumps, infectious mononucleosis, or herpes zoster does not respond to antibiotic therapy and is usually seen in association with characteristic systemic abnormalities. Chronic dacryoadenitis may follow acute inflammation or be due to sarcoidosis or some of the odd inflammatory reactions which merge into the pseudotumour group. It is this group of patients who are difficult to distinguish from those with early malignant tumours arising from the lacrimal gland.

Inflammatory pseudotumours in the region of the lacrimal gland are relatively common. Although they are rare in the very young, the age variation is wide (Chavis et al., 1978). Most commonly the signs and symptoms are similar to those seen in acute dacryoadenitis, though the patient is apyrexial with a normal white cell count. Some patients have a slowly enlarging mass which may not be tender, though the pain is frequently noticed. Plain radiographs are invariably normal, and CT scans will demonstrate the size and extent of the mass. Ultrasonic scans can provide useful information, the pattern of echoes placing the tumour in the lymphoma-pseudotumour group.

Lymphomatous lesions include a broad spectrum from reactive lymphoid hyperplasia to malignant lymphomas of various types. They most commonly occur in adults with a progressive swelling of the eyelid over a period of several months. Inflammatory signs and symptoms are extremely uncommon. A firm nontender mass can usually be palpated in the region of the lacrimal gland. The presence of a purple pink fleshy lesion underneath the conjunctiva of the upper fornix is characteristic of this lesion. The results of investigations are similar to those seen in inflammatory pseudotumours.

Dermoid cysts may also occur in the region of the lacrimal gland and cause some confusion. Peak incidence is in the first decade. Characteristically, 
a smooth mass may be palpated in the upper outer quadrant of the orbit, but in some cases the cyst may lie deeply within the orbit, and the only abnormal physical sign is proptosis. The patients present with a swelling of the upper eyelid or with progressive proptosis. On rare occasions the cyst may become inflamed and produce a similar clinical picture to acute dacryoadenitis. Plain radiographs provide the diagnosis. A sharply defined cyst-like bone defect, sometimes with sclerotic margins, is characteristic. Benign mixed tumours and some malignant lacrimal tumours cause a more generalised expansion of the lacrimal fossa. An invasive carcinoma will produce an ill-defined osteolysis quite unlike the picture of a dermoid. CT scans frequently aid the diagnosis by revealing the cystic nature of the lesion and in some cases a fluid level within the cyst produced by sebaceous material and lipid. Ultrasonic scans should also show the cyst, so that in most cases a preoperative diagnosis is correctly made.

Patients with lacrimal fossa lesions can be conveniently divided into 2 groups on clinical grounds. The first group are patients with a suspected benign mixed tumour which is confined to the lacrimal fossa. These patients have a history of longer than 12 months, radiographic evidence of expansion of the lacrimal fossa, and no history of pain. The second group comprises patients with a variety of lesions, lacrimal gland carcinomas, lymphomas, pseudotumour, and other inflammatory lesions. They have a short history, often with pain, and may or may not have abnormal radiographs.

The importance of recognising the first group on clinical grounds cannot be emphasised too often. The temptation to biopsy the lesion should be avoided, and total removal of the whole of the lacrimal gland through a lateral orbitotomy can be planned. The choice of approach to the gland is of prime importance, for the exposure should be such that the palpebral lobe and the structures of the eyelid can be dissected out under an operating microscope. It is essential that in removing the lacrimal gland plus tumour the capsule of the tumour is not touched and that periosteum overlying the tumour is removed at the same time. The modified lateral orbitotomy approach offers the only way to achieve the correct and adequate removal of the tumour. A transcranial anterior orbitotomy or the Berke lateral orbitotomy makes it extremely difficult or impossible to avoid a subtotal or piecemeal removal of the gland and tumour.

Rupture of the capsule of a benign mixed cell tumour affects the prognosis adversely because of seeding of the tumour cells into the surrounding tissues. Recurrences are thus inevitable, and in many cases, although such recurrences are histologically nonmalignant, malignant changes can occur. In patients in whom the recurrences remain benign the outcome is often indistinguishable from that seen in a true carcinoma. The tumour cells invade the apex of the orbit and the surrounding bone. A painful and lingering death ensues, for in most cases the tumour spreads beyond the line of surgical resection, and, although radiotherapy may be used as a palliative measure, benign mixed cell tumours are relatively radioresistant.

Patients with a very short history of a lacrimal fossa swelling and associated inflammatory signs can reasonably be treated with a short course of antibiotics. Resolution within 2 to 3 weeks would be the expected course. Unless the mass shows a progressive decrease in size, early incisional biopsy should be done. Patients with a history of a few months' swelling in the region of the lacrimal gland with or without inflammatory signs or abnormal $x$-rays should be biopsied forthwith.

One other group of patients can be identified in whom an early biopsy is indicated. These are patients who have a history of longer than 12 months but do not fit into the clinical pattern presented by the benign mixed cell tumours. They often complain of pain and show radiological evidence of invasion of the overlying bone or evidence of calcification within the tumour. A biopsy should be obtained in all these cases through a transseptal incision. The extraperiosteal approach should never be used, for the integrity of the periosteal barrier must be maintained to prevent possible seeding of the extraperiosteal space by malignant cells. By following this regimen a definite diagnosis will be reached at an early stage in the clinical evaluation. Such diverse lesions as inflammatory masses, lymphomas, and carcinomas will be accurately identified and the appropriate treatment started.

Patients found to have adenoid cystic carcinomas and other malignant epithelial neoplasms should be evaluated to determine the extent of the tumour. Where there is evidence of restriction of the tumour mass within the periosteal barrier and without involvement of the orbital apex, a radical resection of the area can be undertaken. The skills of a neurosurgeon and plastic surgeon as well as the of ophthalmic surgeon should be united in such a surgical operation. Surgical resection in these cases should include portions of the lateral and superior orbital walls as well as removal of the lids and orbital contents. A report describing the criteria of operability, recommended surgical approaches, and the results of the experience with malignant lacrimal gland neoplasms at Moorfields Eye Hospital is in 
Table 2 Flowsheet of management of lacrimal gland/fossa masses based on duration of symptoms and radiographic findings

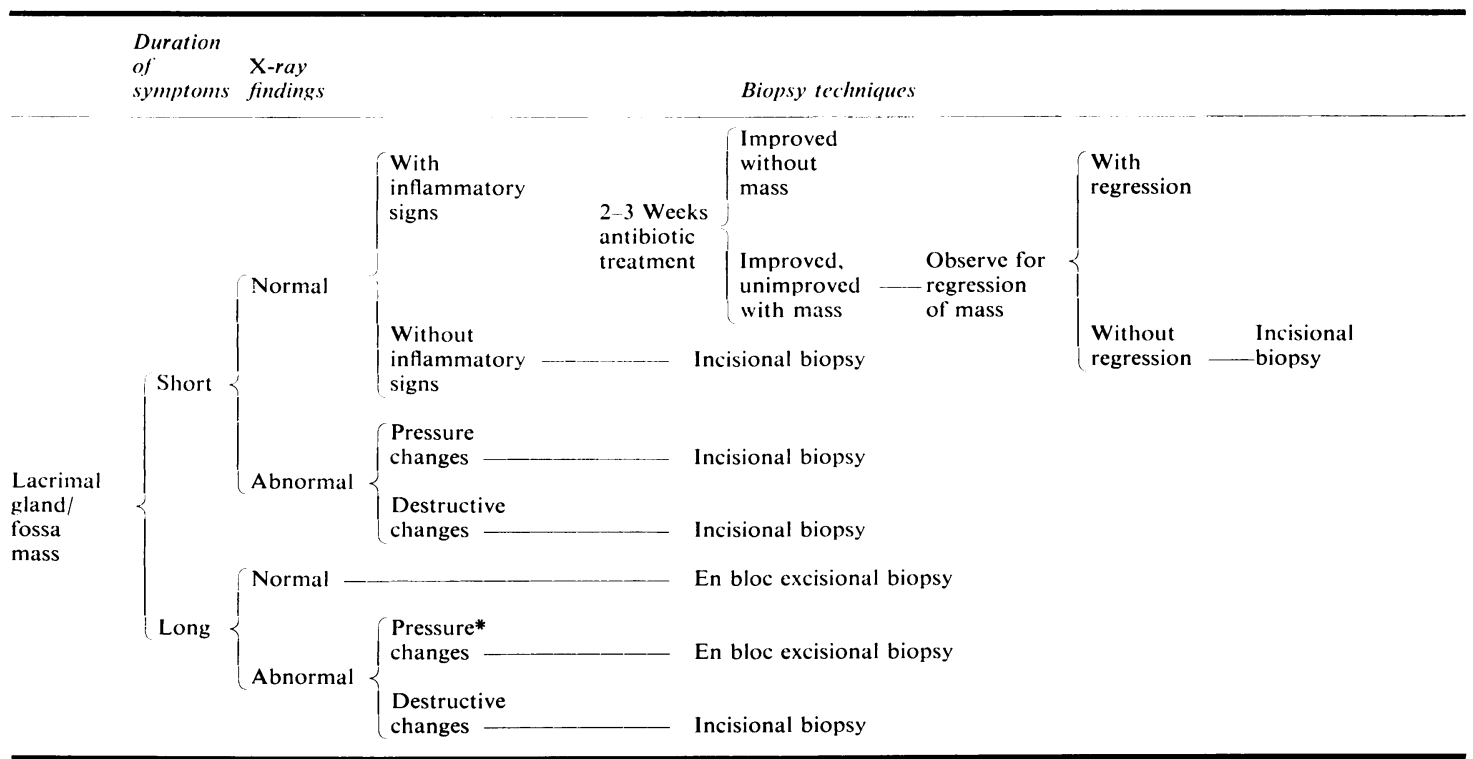

Short $=$ duration less than 12 months. Long $=$ duration greater than 12 months. Incisional biopsies done via trans-septal approach. En bloc excisional biopsy done via lateral orbitotomy.

* Characteristic presentation of benign mixed cell lacrimal gland tumour.

preparation. Radiotherapy and chemotherapy may be considered in those cases in which spread has occurred beyond even these wide surgical margins. The outlook for these patients is extremely poor (Forrest, 1954; Zimmerman et al., 1962; Forrest, 1971; Henderson, 1973; Ashton, 1975).

This approach, graphically summarised in Table 2 , diminishes the need for histological diagnosis by frozen section and permits decisions on extensive surgical removal of a neoplastic lesion to be based on evaluation of permanent appropriately fixed and stained microscopic sections. Furthermore, the risks of incisional biopsy of a benign mixed tumour and its subsequent potential recurrence are minimised. This risk cannot be completely eliminated, for occasionally a patient with a benign mixed-cell tumour arising in the palpebral lobe will present with a short history of a slowly enlarging mass similar to a chalazion, and in these circumstances invariably the lesion is approached directly.

No protocol can provide foolproof solutions to the complex decisions facing the surgeon. Nonetheless, this diagnostic approach based primarily on clinical history and radiographic findings, does provide a logical approach to the problem of lacrimal gland and fossa lesions. In part the dismal prognosis of malignant lacrimal gland tumours is related to delay in diagnosis, with subsequent spread beyond the limits of surgical excision. We think that an improved survival rate can be achieved by an aggressive attitude towards early biopsy followed by extirpative surgery.

Our thanks are due to the various consultant ophthalmic surgeons who referred the cases to the Orbital Clinic, to Dr G. A. S. Lloyd for his help with the interpretation of the radiographs, the Department of Medical Illustration for the preparation of the clinical photographs, and to Mrs S. J. Cole for her assistance in the preparation of the paper.

\section{References}

Ashton, N. L. (1975). Epithelial tumours of the lacrimal gland. Modern Problems of Ophthalmology, 14, 306-323. Karger: Basel.

Chavis, R. M., Garner, A., and Wright, J. E. (1978). Inflammatory orbital pseudotumour. Archives of Ophthalmology, 96, 1817-1822.

Davies, W. S. (1954). Pleomorphic adenoma and adenocarcinoma of the lacrimal gland with report of thirteen cases. Transactions of the American Ophthalmological Society, 52, 467-495.

Duke-Elder, S., and MacFaul, P. A. (1974). The ocular adnexa. In Duke-Elder, S. (ed.), System of Ophthalmology, vol. XIII, pt. 2, pp. 601-622.

Forrest, A. W. (1954). Epithelial lacrimal gland tumours; pathology as a guide to prognosis. Transactions of the American Academy of Ophthalmology and Otolaryngology, 58, 848-866.

Forrest, A. W. (1971). Pathologic criteria for effective management of epithelial lacrimal gland tumours. Ameri- 
can Journal of Ophthalmology, 71, 178-192.

Henderson, J. W. (1973). Orbital Tumours, pp. 409-443. W. B. Saunders: Philadelphia.

Jones, I. S., and Pfeiffer, R. L. (1954). Lacrimal gland tumours: roentgenographic diagnosis. Transactions of the American Academy of Ophthalmology and Otolaryngology, 58, 841-847.

Lloyd, G. A. S. (1975). Radiology of the Orbit, pp. 133-136. W. B. Saunders: London.

Newton, T. H. (1962). Roentgen appearance of lacrimal gland tumours. Radiology, 79, 598-605.

Reese, A. B. (1956). The treatment of expanding lesions of the orbit with particular regard to those arising in the lacrimal gland. American Journal of Ophthalmology, 41, $3-11$.

Rubin, I. E. (1947). Adenocarcinoma of the lacrimal gland following a mixed tumour of 25 years duration. Archives of Ophthalmology, 37, 686-688.

Waller, R., Ridley, F. C., and Henderson, J. W. (1973). Malignant mixed tumour of lacrimal gland, occult source of metastatic carcinoma. Archives of Ophthalmology, 90, 297-299.

Zimmerman, L. E., Sanders, T. E., and Ackerman, L. V. (1962). Epithelial tumours of the lacrimal gland: prognostic and therapeutic significance of histological types. International Ophthalmology Clinics, 2, 337-367. 